\title{
$n$-tuplet fixed point theorems for contractive type mappings in partially ordered metric spaces
}

\author{
Müzeyyen Ertürk ${ }^{1 *}$ and Vatan Karakaya ${ }^{2}$
}

A correction to this article has been published: http://www.journalofinequalitiesandapplications.com/content/2013/1/368.

"Correspondence:

merturk3263@gmail.com; merturk@yildiz.edu.tr

'Department of Mathematics, The Faculty of Arts and Sciences, Yildiz Technical University, Davutpasa Campus, Esenler, Istanbul, 34210, Turkey

Full list of author information is available at the end of the article

\begin{abstract}
In this paper, we study existence and uniquennes of fixed points of operator $F: X^{n} \rightarrow X$ where $n$ is an arbitrary positive integer and $X$ is partially ordered complete metric space.

MSC: Primary 47H10; 54H25; secondary 54E50

Keywords: fixed point theorems; nonlinear contraction; partially ordered metric space; $n$-tuplet fixed point; mixed $g$-monotone
\end{abstract}

\section{Introduction}

As it is known, fixed point theory is one of the oldest and most famous theory in mathematics, and it has become an important tool for other areas of science such as approximation theory, statistics, engineering and economics.

Among hundreds of fixed point theorems, the Banach contraction theorem [1] is particularly well known due to its simplicity and usefulness. It states that any contraction mapping of a complete metric space has a unique fixed point.

In 2004, the Banach contraction principle were extended to metric space endowed with partial order by Ran and Reuring [2]. They pointed out that the contractivity condition on the nonlinear and monotone map is only assumed to hold on elements which are comparable in the partial order. Afterward, Nieto and Rodriguez-Lopez [3] extended results of Ran and Reuring for non-decreasing mapping and studied existence and uniqueness of first-order differential equations.

In 2006, by following the above mentioned trend, Bhaskar and Lakshmikantham [4] introduced mixed monotone property and gave their coupled fixed point theorem for mappings with mixed monotone property. Also, they produced some applications related with the existence and uniqueness of solution for a periodic boundary value problem. This work of Bhaskar and Lakshmikantham has attracted the attention of many researchers. The concept of coupled fixed point for various contractive type mappings was studied by several authors [5-10]. Lakshmikantham and Ciric [11] extended the results of [4] for monotone non-linear contractive mapping and generalized mixed monotone concept. Berinde and Borcut [12] introduced tripled fixed point theorem for non-linear mapping in partially or- 
dered complete metric space as a generalization and extension of the coupled fixed point theorem.

Motivated by these studies, the quadruple fixed point theorem was given for different contractive type mappings [13-16].

In this paper, we generalize mentioned trend in the above for an arbitrary positive number $n$, that is, we introduce the concept of $n$-tuplet fixed point theorem and prove some results.

\section{Main results}

Let us give new definitions for our aim.

Definition 1 Let $(X, \leq)$ be partially ordered set and $F: X^{n} \rightarrow X$. We say that $F$ has the mixed monotone property if $F\left(x_{1}, x_{2}, x_{3}, \ldots, x_{n}\right)$ is monotone non-decreasing in its odd argument and it is monotone non-increasing in its even argument. That is, for any $x_{1}, x_{2}, x_{3}, \ldots, x_{n} \in X$

$$
\begin{aligned}
& y_{1}, z_{1} \in X, \quad y_{1} \leq z_{1} \\
& \quad \Longrightarrow \quad F\left(y_{1}, x_{2}, x_{3}, \ldots, x_{n}\right) \leq F\left(z_{1}, x_{2}, x_{3}, \ldots, x_{n}\right), \\
& y_{2}, z_{2} \in X, \quad y_{2} \leq z_{2} \\
& \quad \Longrightarrow \quad F\left(x_{1}, y_{2}, x_{3}, \ldots, x_{n}\right) \geq F\left(x_{1}, z_{2}, x_{3}, \ldots, x_{n}\right), \\
& \vdots \\
& y_{n}, z_{n} \in X, \quad y_{n} \leq z_{n} \\
& \quad \Longrightarrow \quad F\left(x_{1}, x_{2}, x_{3}, \ldots, y_{n}\right) \leq F\left(x_{1}, x_{2}, x_{3}, \ldots, z_{n}\right) \quad \text { (if } n \text { is odd) } \\
& y_{n}, z_{n} \in X, \quad y_{n} \leq z_{n} \\
& \quad \Longrightarrow \quad F\left(x_{1}, x_{2}, x_{3}, \ldots, y_{n}\right) \geq F\left(x_{1}, x_{2}, x_{3}, \ldots, z_{n}\right) \quad \text { (if } n \text { is even). }
\end{aligned}
$$

Definition 2 Let $X$ be a nonempty set and $F: X^{n} \rightarrow X$ a given mapping. An element $\left(x_{1}, x_{2}, x_{3}, \ldots, x_{n}\right) \in X^{n}$ is called a $n$-tuplet fixed point of $F$ if

$$
\begin{aligned}
& F\left(x_{1}, x_{2}, x_{3}, \ldots, x_{n}\right)=x_{1}, \\
& F\left(x_{2}, x_{3}, \ldots, x_{n}, x_{1}\right)=x_{2}, \\
& \vdots \\
& F\left(x_{n}, x_{1}, x_{2}, \ldots, x_{n-1}\right)=x_{n} .
\end{aligned}
$$

Definition 3 Let $(X, \leq)$ be partially ordered set and $F: X^{n} \rightarrow X$ and $g: X \rightarrow X$. We say that $F$ has the mixed $g$-monotone property if $F\left(x_{1}, x_{2}, x_{3}, \ldots, x_{n}\right)$ is monotone $g$-non-decreasing in its odd argument and it is monotone $g$-non-increasing in its even argument. That is, for any $x_{1}, x_{2}, x_{3}, \ldots, x_{n} \in X$

$$
\begin{aligned}
& y_{1}, z_{1} \in X, \quad g\left(y_{1}\right) \leq g\left(z_{1}\right) \\
& \quad \Longrightarrow \quad F\left(y_{1}, x_{2}, x_{3}, \ldots, x_{n}\right) \leq F\left(z_{1}, x_{2}, x_{3}, \ldots, x_{n}\right), \\
& y_{2}, z_{2} \in X, \quad g\left(y_{2}\right) \leq g\left(z_{2}\right) \\
& \quad \Longrightarrow \quad F\left(x_{1}, y_{2}, x_{3}, \ldots, x_{n}\right) \geq F\left(x_{1}, z_{2}, x_{3}, \ldots, x_{n}\right),
\end{aligned}
$$




$$
\begin{aligned}
& y_{n}, z_{n} \in X, \quad g\left(y_{n}\right) \leq g\left(z_{n}\right) \\
& \Longrightarrow \quad F\left(x_{1}, x_{2}, x_{3}, \ldots, y_{n}\right) \leq F\left(x_{1}, x_{2}, x_{3}, \ldots, z_{n}\right) \quad \text { (if } n \text { is odd) } \\
& y_{n}, z_{n} \in X, \quad g\left(x_{n}\right) \leq g\left(y_{n}\right) \\
& \Longrightarrow \quad F\left(x_{1}, x_{2}, x_{3}, \ldots, y_{n}\right) \geq F\left(x_{1}, x_{2}, x_{3}, \ldots, z_{n}\right) \quad \text { (if } n \text { is even). }
\end{aligned}
$$

Note that if $g$ is the identity mapping, this definition reduces to Definition 1.

Definition 4 Let $X$ be a nonempty set and $F: X^{n} \rightarrow X$ a given mapping. An element $\left(x_{1}, x_{2}, x_{3}, \ldots, x_{n}\right) \in X^{n}$ is called a $n$-tuplet coincidence point of $F: X^{n} \rightarrow X$ and $g: X \rightarrow X$ if

$$
\begin{aligned}
& F\left(x_{1}, x_{2}, x_{3}, \ldots, x_{n}\right)=g\left(x_{1}\right), \\
& F\left(x_{2}, x_{3}, \ldots, x_{n}, x_{1}\right)=g\left(x_{2}\right), \\
& \vdots \\
& F\left(x_{n}, x_{1}, x_{2}, \ldots, x_{n-1}\right)=g\left(x_{n}\right) .
\end{aligned}
$$

Note that if $g$ is the identity mapping, this definition reduces to Definition 2.

Definition 5 Let $(X, \leq)$ be partially ordered set and $F: X^{n} \rightarrow X$ and $g: X \rightarrow X$.F and $g$ called commutative if

$$
g\left(F\left(x_{1}, x_{2}, x_{3}, \ldots, x_{n}\right)\right)=F\left(g\left(x_{1}\right), g\left(x_{2}\right), g\left(x_{3}\right), \ldots, g\left(x_{n}\right)\right)
$$

for all $x_{1}, x_{2}, x_{3}, \ldots, x_{n} \in X$.

Let $\Phi$ denote the all functions $\phi:[0, \infty) \rightarrow[0, \infty)$, which are continuous and satisfy that

(i) $\phi(t)<t$,

(ii) $\lim _{r \rightarrow t_{+}} \phi(r)<t$ for each $r>0$.

Since we want to shorten expressions in the following theorem, consider Condition 1 in the following for $X$ an $F$.

Condition 1 Suppose either

(i) $F$ is continuous, or

(ii) $X$ has the following property:

(a) if non-decreasing sequence $x_{k} \rightarrow x$, then $x_{k} \leq x$ for all $k$,

(b) if non-increasing sequence $y_{k} \rightarrow y$, then $y_{k} \geq y$ for all $k$.

Theorem 1 Let $(X, \leq)$ be partially ordered set and suppose that $(X, d)$ is complete metric space. Assume $F: X^{n} \rightarrow X$ and $g: X \rightarrow X$ are such that $F$ has the mixed $g$-monotone property and

$$
\begin{aligned}
& d\left(F\left(x_{1}, x_{2}, x_{3}, \ldots, x_{n}\right), F\left(y_{1}, y_{2}, y_{3}, \ldots, y_{n}\right)\right) \\
& \quad \leq \phi\left(\frac{d\left(g\left(x_{1}\right), g\left(y_{1}\right)\right)+d\left(g\left(x_{2}\right), g\left(y_{2}\right)\right)+\cdots+d\left(g\left(x_{n}\right), g\left(y_{n}\right)\right)}{n}\right)
\end{aligned}
$$


for all $x_{1}, x_{2}, x_{3}, \ldots, x_{n} \in X$ for which $g\left(x_{1}\right) \leq g\left(y_{1}\right), g\left(x_{2}\right) \geq g\left(y_{2}\right), \ldots, g\left(x_{n}\right) \leq g\left(y_{n}\right)$ (if $n$ is odd), $g\left(x_{n}\right) \geq g\left(y_{n}\right)$ (if $n$ is even). Assume that $F\left(X^{n}\right) \subset g(X)$ and $g$ commutes with $F$. Also, suppose that Condition 1 is satisfied. If there exist $x_{0}^{1}, x_{0}^{2}, x_{0}^{3}, \ldots, x_{0}^{n} \in X$ such that

$$
\begin{aligned}
& g\left(x_{0}^{1}\right) \leq F\left(x_{0}^{1}, x_{0}^{2}, x_{0}^{3}, \ldots, x_{0}^{n}\right), \\
& g\left(x_{0}^{2}\right) \geq F\left(x_{0}^{2}, x_{0}^{3}, \ldots, x_{0}^{n}, x_{0}^{1}\right), \\
& \vdots \\
& g\left(x_{0}^{n}\right) \leq F\left(x_{0}^{n}, x_{0}^{1}, x_{0}^{2}, \ldots, x_{0}^{n-1}\right) \quad(\text { if } n \text { is odd }), \\
& g\left(x_{0}^{n}\right) \geq F\left(x_{0}^{n}, x_{0}^{1}, x_{0}^{2}, \ldots, x_{0}^{n-1}\right) \quad(\text { if } n \text { is even })
\end{aligned}
$$

then there exist $x_{1}, x_{2}, x_{3}, \ldots, x_{n} \in X$ such that

$$
\begin{aligned}
& F\left(x_{1}, x_{2}, x_{3}, \ldots, x_{n}\right)=g\left(x_{1}\right), \\
& F\left(x_{2}, x_{3}, \ldots, x_{n}, x_{1}\right)=g\left(x_{2}\right), \\
& \vdots \\
& F\left(x_{n}, x_{1}, x_{2}, \ldots, x_{n-1}\right)=g\left(x_{n}\right),
\end{aligned}
$$

that is, $F$ and $g$ have a $n$-tuplet coincidence point.

Proof Let $x_{0}^{1}, x_{0}^{2}, x_{0}^{3}, \ldots, x_{0}^{n} \in X$ be such that (2.7). Since $F\left(X^{n}\right) \subset g(X)$, we construct the sequence $\left(x_{k}^{1}\right),\left(x_{k}^{2}\right), \ldots,\left(x_{k}^{n}\right)$ as follows:

$$
\begin{aligned}
& g\left(x_{k}^{1}\right)=F\left(x_{k-1}^{1}, x_{k-1}^{2}, \ldots, x_{k-1}^{n}\right), \\
& g\left(x_{k}^{2}\right)=F\left(x_{k-1}^{2}, \ldots, x_{k-1}^{n}, x_{k-1}^{1}\right), \\
& \vdots \\
& g\left(x_{k}^{n}\right)=F\left(x_{k-1}^{n}, x_{k-1}^{1}, \ldots, x_{k-1}^{n-1}\right)
\end{aligned}
$$

for $k=1,2,3, \ldots$. We claim that

$$
\begin{aligned}
& g\left(x_{k-1}^{1}\right) \leq g\left(x_{k}^{1}\right), \\
& g\left(x_{k-1}^{2}\right) \geq g\left(x_{k}^{2}\right), \\
& \vdots \\
& g\left(x_{k-1}^{n}\right) \leq g\left(x_{k}^{n}\right) \quad \text { (if } n \text { is odd), } \\
& g\left(x_{k-1}^{n}\right) \geq g\left(x_{k}^{n}\right) \quad(\text { if } n \text { is even })
\end{aligned}
$$

for all $k \geq 1$. For this, we will use the mathematical induction. The inequalities in (2.9) hold $k=1$ because of (2.7), that is, we have

$$
\begin{aligned}
& g\left(x_{0}^{1}\right) \leq F\left(x_{0}^{1}, x_{0}^{2}, \ldots, x_{0}^{n}\right)=g\left(x_{1}^{1}\right), \\
& g\left(x_{0}^{2}\right) \geq F\left(x_{0}^{2}, \ldots, x_{0}^{n}, x_{0}^{1}\right)=g\left(x_{1}^{2}\right),
\end{aligned}
$$




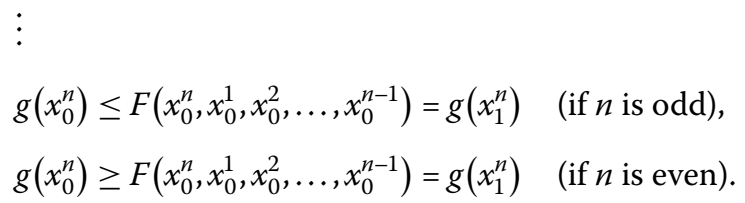

Thus, our claim is true for $k=1$. Now, suppose that the inequalities in (2.9) hold $k=m$. In this case,

$$
\begin{aligned}
& g\left(x_{m-1}^{1}\right) \leq g\left(x_{m}^{1}\right), \\
& g\left(x_{m-1}^{2}\right) \geq g\left(x_{m}^{2}\right), \\
& \vdots \\
& g\left(x_{m-1}^{n}\right) \leq g\left(x_{m}^{n}\right) \quad \text { (if } n \text { is odd), } \\
& g\left(x_{m-1}^{n}\right) \geq g\left(x_{m}^{n}\right) \quad \text { (if } n \text { is even). }
\end{aligned}
$$

Now, we must show that the inequalities in (2.9) hold $k=m+1$. If we consider (2.8) and mixed $g$-monotone property of $F$ together with (2.10), we have

$$
\begin{aligned}
g\left(x_{m}^{1}\right) & =F\left(x_{m-1}^{1}, x_{m-1}^{2}, \ldots, x_{m-1}^{n}\right) \\
& \leq F\left(x_{m}^{1}, x_{m-1}^{2}, \ldots, x_{m-1}^{n}\right) \\
& \leq F\left(x_{m}^{1}, x_{m}^{2}, x_{m-1}^{3}, \ldots, x_{m-1}^{n-1}, x_{m-1}^{n}\right) \\
& \vdots \\
& \leq F\left(x_{m}^{1}, x_{m}^{2}, \ldots, x_{m}^{n-1}, x_{m-1}^{n}\right) \\
& \leq F\left(x_{m}^{1}, x_{m}^{2}, \ldots, x_{m}^{n}\right)=g\left(x_{m+1}^{1}\right), \\
g\left(x_{m}^{2}\right) & =F\left(x_{m-1}^{2}, \ldots, x_{m-1}^{n}, x_{m-1}^{1}\right) \\
& \geq F\left(x_{m}^{2}, x_{m-1}^{3}, \ldots, x_{m-1}^{n}, x_{m-1}^{1}\right) \\
& \geq F\left(x_{m}^{2}, x_{m}^{3}, \ldots, x_{m-1}^{n}, x_{m-1}^{1}\right) \\
& \vdots \\
& \geq F\left(x_{m}^{2}, x_{m}^{3}, \ldots, x_{m}^{n}, x_{m-1}^{1}\right) \\
& \geq F\left(x_{m}^{2}, x_{m}^{3}, \ldots, x_{m}^{n}, x_{m}^{1}\right)=g\left(x_{m+1}^{2}\right), \\
\vdots & \\
g\left(x_{m}^{n}\right) & =F\left(x_{m-1}^{n}, x_{m-1}^{1}, \ldots, x_{m-1}^{n-1}\right) \\
& \leq F\left(x_{m}^{n}, x_{m-1}^{1}, \ldots, x_{m-1}^{n-1}\right) \\
& \leq F\left(x_{m}^{n}, x_{m}^{1}, x_{m-1}^{2}, \ldots, x_{m-1}^{n-1}\right) \\
& \vdots \\
&
\end{aligned}
$$




$$
\begin{aligned}
& \left.\leq F\left(x_{m}^{n}, x_{m}^{1}, \ldots, x_{m}^{n-1}\right)=g\left(x_{m+1}^{n}\right) \quad \text { (if } n \text { is odd }\right), \\
g\left(x_{m}^{n}\right) & =F\left(x_{m-1}^{n}, x_{m-1}^{1}, \ldots, x_{m-1}^{n-1}\right) \\
& \geq F\left(x_{m}^{n}, x_{m-1}^{1}, \ldots, x_{m-1}^{n-1}\right) \\
& \geq F\left(x_{m}^{n}, x_{m}^{1}, x_{m-1}^{2}, \ldots, x_{m-1}^{n-1}\right) \\
& \vdots \\
& \geq F\left(x_{m}^{n}, x_{m}^{1}, x_{m}^{2}, \ldots, x_{m}^{n-2}, x_{m-1}^{n-1}\right) \\
& \left.\geq F\left(x_{m}^{n}, x_{m}^{1}, \ldots, x_{m}^{n-1}\right)=g\left(x_{m+1}^{n}\right) \quad \text { (if } n \text { is even }\right) .
\end{aligned}
$$

Thus, (2.9) is satisfied for all $k \geq 1$. So, we have,

$$
\begin{aligned}
& \cdots \geq g\left(x_{k}^{1}\right) \geq g\left(x_{k-1}^{1}\right) \geq \cdots \geq g\left(x_{1}^{1}\right) \geq g\left(x_{0}^{1}\right), \\
& \cdots \leq g\left(x_{k}^{2}\right) \leq g\left(x_{k-1}^{2}\right) \leq \cdots \leq g\left(x_{1}^{2}\right) \leq g\left(x_{0}^{2}\right),
\end{aligned}
$$

$\vdots$

$$
\begin{aligned}
& \cdots \geq g\left(x_{k}^{n}\right) \geq g\left(x_{k-1}^{n}\right) \geq \cdots \geq g\left(x_{1}^{n}\right) \geq g\left(x_{0}^{n}\right) \quad(\text { if } n \text { is odd }) \\
& \cdots \leq g\left(x_{k}^{n}\right) \leq g\left(x_{k-1}^{n}\right) \leq \cdots \leq g\left(x_{1}^{n}\right) \leq g\left(x_{0}^{n}\right) \quad(\text { if } n \text { is even })
\end{aligned}
$$

For the simplicity, we define

$$
\delta_{k}=d\left(g\left(x_{k}^{1}\right), g\left(x_{k+1}^{1}\right)\right)+d\left(g\left(x_{k}^{2}\right), g\left(x_{k+1}^{2}\right)\right)+\cdots+d\left(g\left(x_{k}^{n}\right), g\left(x_{k+1}^{n}\right)\right) .
$$

We will show that

$$
\delta_{k+1} \leq n \cdot \phi\left(\frac{\delta_{k}}{n}\right)
$$

By (2.6), (2.8) and (2.11), we get

$$
\begin{aligned}
& d\left(g\left(x_{k+1}^{1}\right), g\left(x_{k+2}^{1}\right)\right) \\
& \quad=d\left(F\left(x_{k}^{1}, x_{k}^{2}, \ldots, x_{k}^{n}\right), F\left(x_{k+1}^{1}, x_{k+1}^{2}, \ldots, x_{k+1}^{n}\right)\right) \\
& \quad \leq \phi\left(\frac{d\left(g\left(\left(x_{k}^{1}\right), g\left(x_{k+1}^{1}\right)\right)\right)+d\left(g\left(x_{k}^{2}\right), g\left(x_{k+1}^{2}\right)\right)+\cdots+d\left(g\left(x_{k}^{n}\right), g\left(x_{k+1}^{n}\right)\right)}{n}\right) \\
& \quad=\phi\left(\frac{\delta_{k}}{n}\right), \\
& d\left(g\left(x_{k+1}^{2}\right), g\left(x_{k+2}^{2}\right)\right) \\
& \quad=d\left(F\left(x_{k}^{2}, \ldots, x_{k}^{n}, x_{k}^{1}\right), F\left(x_{k+1}^{2}, \ldots, x_{k+1}^{n}, x_{k+1}^{1}\right)\right) \\
& \quad \leq \phi\left(\frac{d\left(g\left(x_{k}^{2}\right), g\left(x_{k+1}^{2}\right)\right)+\cdots+d\left(g\left(x_{k}^{n}\right), g\left(x_{k+1}^{n}\right)\right)+d\left(g\left(x_{k}^{1}\right), g\left(x_{k+1}^{1}\right)\right)}{n}\right) \\
& \quad=\phi\left(\frac{\delta_{k}}{n}\right),
\end{aligned}
$$




$$
\begin{aligned}
& d\left(g\left(x_{k}^{n}\right), g\left(x_{k+2}^{n}\right)\right) \\
& \quad=d\left(F\left(x_{k}^{n}, x_{k}^{1}, \ldots, x_{k}^{n-1}\right), F\left(x_{k+1}^{n}, x_{k+1}^{1}, \ldots, x_{k+1}^{n-1}\right)\right) \\
& \quad \leq \phi\left(\frac{d\left(g\left(\left(x_{k}^{n}\right), g\left(x_{k+1}^{n}\right)\right)\right)+d\left(g\left(x_{k}^{1}\right), g\left(x_{k+1}^{1}\right)\right)+\cdots+d\left(g\left(x_{k}^{n-1}\right), g\left(x_{k+1}^{n-1}\right)\right)}{n}\right) \\
& \quad=\phi\left(\frac{\delta_{k}}{n}\right) .
\end{aligned}
$$

Due to (2.13)-(2.15), we conclude that

$$
\begin{aligned}
& d\left(g\left(x_{k+1}^{1}\right), g\left(x_{k+2}^{1}\right)\right)+d\left(g\left(x_{k+1}^{2}\right), g\left(x_{k+2}^{2}\right)\right)+\cdots \\
& \quad+d\left(g\left(x_{k+1}^{n}\right), g\left(x_{k+2}^{n}\right)\right) \leq n \phi\left(\frac{\delta_{k}}{n}\right) .
\end{aligned}
$$

Hence, we get (2.12).

Since $\phi(t)<t$ for all $t>0$, then $\delta_{k+1} \leq n \phi\left(\frac{\delta_{k}}{n}\right)<n \cdot \frac{\delta_{k}}{n}=\delta_{k}$ for all $k \in \mathbb{N}$. So, $\left(\delta_{k}\right)$ is monotone decreasing. Since it is bounded below, there is some $\delta \geq 0$ such that

$$
\lim _{k \rightarrow \infty} \delta_{k}=\delta+
$$

We want to show that $\delta=0$. Suppose that $\delta>0$. Then taking the limit as $\delta_{k} \rightarrow \delta+$ of both sides of (2.12) and keeping in mind that we assume that $\lim _{r \rightarrow t_{+}} \phi(r)<t$ for all $t>0$, we have

$$
\delta=\lim _{k \rightarrow \infty} \delta_{k+1} \leq \lim _{k \rightarrow \infty} n \cdot \phi\left(\frac{\delta_{k}}{n}\right)=n \lim _{\delta_{k} \rightarrow \delta+} \phi\left(\frac{\delta_{k}}{n}\right)<n \frac{\delta}{n}=\delta
$$

which is a contradiction. Thus, $\delta=0$, that is

$$
\lim _{k \rightarrow \infty}\left(d\left(g\left(x_{k}^{1}\right), g\left(x_{k+1}^{1}\right)\right)+d\left(g\left(x_{k}^{2}\right), g\left(x_{k+1}^{2}\right)\right)+\cdots+d\left(g\left(x_{k}^{n}\right), g\left(x_{k+1}^{n}\right)\right)\right)=0 .
$$

Now we prove that $g\left(x_{k}^{1}\right), g\left(x_{k}^{2}\right), \ldots, g\left(x_{k}^{n}\right)$ are Cauchy sequences. Suppose that at least one of $g\left(x_{k}^{1}\right), g\left(x_{k}^{2}\right), \ldots, g\left(x_{k}^{n}\right)$ is not Cauchy. So, there exists an $\varepsilon>0$ for which we can find subsequence of integer $j(k), l(k)$ with $j(k)>l(k) \geq k$ such that

$$
t_{k}=d\left(g\left(x_{j(k)}^{1}\right), g\left(x_{l(k)}^{1}\right)\right)+d\left(g\left(x_{j(k)}^{2}\right), g\left(x_{l(k)}^{2}\right)\right)+\cdots+d\left(g\left(x_{j(k)}^{n}\right), g\left(x_{l(k)}^{n}\right)\right) \geq \varepsilon .
$$

Additionally, corresponding to $j(k)$, we can choose $l(k)$ such that it is the smallest integer satisfying $(2.20)$ and $j(k)>l(k) \geq k$. Thus,

$$
d\left(g\left(x_{j(k)-1}^{1}\right), g\left(x_{l(k)}^{1}\right)\right)+d\left(g\left(x_{j(k)-1}^{2}\right), g\left(x_{l(k)}^{2}\right)\right)+\cdots+d\left(g\left(x_{j(k)-1}^{n}\right), g\left(x_{l(k)}^{n}\right)\right)<\varepsilon .
$$

By using triangle inequality and having (2.20) and (2.21) in mind

$$
\begin{aligned}
\varepsilon & \leq t_{k} \\
& =d\left(g\left(x_{j(k)}^{1}\right), g\left(x_{l(k)}^{1}\right)\right)+d\left(g\left(x_{j(k)}^{2}\right), g\left(x_{l(k)}^{2}\right)\right)+\cdots+d\left(g\left(x_{j(k)}^{n}\right), g\left(x_{l(k)}^{n}\right)\right)
\end{aligned}
$$




$$
\begin{aligned}
\leq & d\left(g\left(x_{j(k)}^{1}\right), g\left(x_{j(k)-1}^{1}\right)\right)+d\left(g\left(x_{j(k)-1}^{1}\right), g\left(x_{l(k)}^{1}\right)\right) \\
& +d\left(g\left(x_{j(k)}^{2}\right), g\left(x_{j(k)-1}^{2}\right)\right)+d\left(g\left(x_{j(k)-1}^{2}\right), g\left(x_{l(k)}^{2}\right)\right) \\
& +\cdots+d\left(g\left(x_{j(k)}^{n}\right), g\left(x_{j(k)-1}^{n}\right)\right)+d\left(g\left(x_{j(k)-1}^{n}\right), g\left(x_{l(k)}^{n}\right)\right) \\
< & d\left(g\left(x_{j(k)}^{1}\right), g\left(x_{j(k)-1}^{1}\right)\right)+d\left(g\left(x_{j(k)}^{2}\right), g\left(x_{j(k)-1}^{2}\right)\right) \\
& +\cdots+d\left(g\left(x_{j(k)}^{n}\right), g\left(x_{j(k)-1}^{n}\right)\right)+\varepsilon \\
< & \delta_{j(k)-1}+\varepsilon .
\end{aligned}
$$

Letting $k \rightarrow \infty$ in (2.22) and using (2.20)

$$
\begin{aligned}
\lim _{k \rightarrow \infty} t_{k} & =\lim _{k \rightarrow \infty}\left[d\left(g\left(x_{j(k)}^{1}\right), g\left(x_{l(k)}^{1}\right)\right)+d\left(g\left(x_{j(k)}^{2}\right), g\left(x_{l(k)}^{2}\right)\right)+\cdots+d\left(g\left(x_{j(k)}^{n}\right), g\left(x_{l(k)}^{n}\right)\right)\right] \\
& =\varepsilon+.
\end{aligned}
$$

We apply triangle inequality to $(2.20)$ as the following.

$$
\begin{aligned}
t_{k}= & d\left(g\left(x_{j(k)}^{1}\right), g\left(x_{l(k)}^{1}\right)\right)+d\left(g\left(x_{j(k)}^{2}\right), g\left(x_{l(k)}^{2}\right)\right) \\
& +\cdots+d\left(g\left(x_{j(k)}^{n}\right), g\left(x_{l(k)}^{n}\right)\right) \\
\leq & d\left(g\left(x_{j(k)}^{1}\right), g\left(x_{j(k)+1}^{1}\right)\right)+d\left(g\left(x_{j(k)+1}^{1}\right), g\left(x_{l(k)+1}^{1}\right)\right)+d\left(g\left(x_{l(k)+1}^{1}\right), g\left(x_{l(k)}^{1}\right)\right) \\
& +d\left(g\left(x_{j(k)}^{2}\right), g\left(x_{j(k)+1}^{2}\right)\right)+d\left(g\left(x_{j(k)+1}^{2}\right), g\left(x_{l(k)+1}^{2}\right)\right)+d\left(g\left(x_{l(k)+1}^{2}\right), g\left(x_{l(k)}^{2}\right)\right) \\
& +\cdots+d\left(g\left(x_{j(k)}^{n}\right), g\left(x_{j(k)+1}^{n}\right)\right)+d\left(g\left(x_{j(k)+1}^{n}\right), g\left(x_{l(k)+1}^{n}\right)\right)+d\left(g\left(x_{l(k)+1}^{n}\right), g\left(x_{l(k)}^{n}\right)\right) \\
\leq & \delta_{j(k)+1}+\delta_{l(k)+1}+d\left(g\left(x_{j(k)+1}^{1}\right), g\left(x_{l(k)+1}^{1}\right)\right)+d\left(g\left(x_{j(k)+1}^{2}\right), g\left(x_{l(k)+1}^{2}\right)\right) \\
& +\cdots+d\left(g\left(x_{j(k)+1}^{n}\right), g\left(x_{l(k)+1}^{n}\right)\right) .
\end{aligned}
$$

Since $j(k)>l(k)$, then

$$
\begin{aligned}
& g\left(x_{j(k)}^{1}\right) \geq g\left(x_{l(k)}^{1}\right), \\
& g\left(x_{j(k)}^{2}\right) \leq g\left(x_{l(k)}^{2}\right), \\
& \vdots \\
& g\left(x_{j(k)}^{n}\right) \geq g\left(x_{l(k)}^{n}\right) \quad \text { (if } n \text { is odd), } \\
& g\left(x_{j(k)}^{n}\right) \leq g\left(x_{l(k)}^{n}\right) \quad \text { (if } n \text { is even). }
\end{aligned}
$$

So, from (2.25), (2.8) and (2.6), we get

$$
\begin{aligned}
& d\left(g\left(x_{j(k)+1}^{1}\right), g\left(x_{l(k)+1}^{1}\right)\right) \\
& \quad=d\left(F\left(x_{j(k)}^{1}, x_{j(k)}^{2}, \ldots, x_{j(k)}^{n}\right), F\left(x_{l(k)}^{1}, x_{l(k)}^{2}, \ldots, x_{l(k)}^{n}\right)\right) \\
& \quad \leq \phi\left(\frac{1}{n}\left[d\left(g\left(x_{j(k)}^{1}\right), g\left(x_{l(k)}^{1}\right)\right)+d\left(g\left(x_{j(k)}^{2}\right), g\left(x_{l(k)}^{2}\right)\right)+\cdots+d\left(g\left(x_{j(k)}^{n}\right), g\left(x_{l(k)}^{n}\right)\right)\right]\right) \\
& \quad=\phi\left(\frac{t_{k}}{n}\right),
\end{aligned}
$$




$$
\begin{aligned}
& d\left(g\left(x_{j(k)+1}^{2}\right), g\left(x_{l(k)+1}^{2}\right)\right) \\
& \quad=d\left(F\left(x_{j(k)}^{2}, \ldots, x_{j(k)}^{n}, x_{j(k)}^{1}\right), F\left(x_{l(k)}^{2}, \ldots, x_{l(k)}^{n}, x_{l(k)}^{1}\right)\right) \\
& \quad \leq \phi\left(\frac{1}{n}\left[d\left(g\left(x_{j(k)}^{2}\right), g\left(x_{l(k)}^{2}\right)\right)+\cdots+d\left(g\left(x_{j(k)}^{n}\right), g\left(x_{l(k)}^{n}\right)\right)+d\left(g\left(x_{j(k)}^{1}\right), g\left(x_{l(k)}^{1}\right)\right)\right]\right) \\
& \quad=\phi\left(\frac{t_{k}}{n}\right), \\
& \quad \vdots \\
& \quad=d\left(g\left(x_{j(k)+1}^{n}\right), g\left(x_{l(k)+1}^{n}\right)\right) \\
& \left.\quad \leq \phi\left(\frac{1}{n}\left[d\left(g\left(x_{j(k)}^{n}, x_{j(k)}^{1}, \ldots, x_{j(k)}^{n-1}\right), F\left(x_{l(k)}^{n}\right)\right)+d\left(g\left(x_{l(k)}^{n}, x_{l(k)}^{1}, \ldots, x_{l(k)}^{n-1}\right)\right), g\left(x_{l(k)}^{1}\right)\right)+\cdots+d\left(g\left(x_{j(k)}^{n-1}\right), g\left(x_{l(k)}^{n-1}\right)\right)\right]\right) \\
& \quad=\phi\left(\frac{t_{k}}{n}\right) .
\end{aligned}
$$

Combining (2.24) with (2.26)-(2.29), we get

$$
\begin{aligned}
t_{k} \leq & \delta_{j(k)+1}+\delta_{l(k)+1} \\
& +d\left(g\left(x_{j(k)+1}^{1}\right), g\left(x_{l(k)+1}^{1}\right)\right)+d\left(g\left(x_{k(k)+1}^{2}\right), g\left(x_{l(k)+1}^{2}\right)\right) \\
& +\cdots+d\left(g\left(x_{j(k)+1}^{n}\right), g\left(x_{l(k)+1}^{n}\right)\right) \\
\leq & \delta_{j(k)+1}+\delta_{l(k)+1}+t_{k}+n \cdot \phi\left(\frac{t_{k}}{n}\right) \\
< & \delta_{j(k)+1}+\delta_{l(k)+1}+n \cdot \frac{t_{k}}{n} .
\end{aligned}
$$

Letting $k \rightarrow \infty$, we obtain a contradiction. This show that $g\left(x_{k}^{1}\right), g\left(x_{k}^{2}\right), \ldots, g\left(x_{k}^{n}\right)$ are Cauchy sequences. Since $X$ is complete metric space, there exist $x^{1}, x^{2}, \ldots, x^{n} \in X$ such that

$$
\lim _{k \rightarrow \infty} g\left(x_{k}^{1}\right)=x^{1}, \quad \lim _{k \rightarrow \infty} g\left(x_{k}^{2}\right)=x^{2}, \quad \ldots, \quad \lim _{k \rightarrow \infty} g\left(x_{k}^{n}\right)=x^{n}
$$

Since $g$ is continuous, (2.30) implies that

$$
\begin{aligned}
& \lim _{k \rightarrow \infty} g\left(g\left(x_{k}^{1}\right)\right)=g\left(x^{1}\right), \quad \lim _{k \rightarrow \infty} g\left(g\left(x_{k}^{2}\right)\right)=g\left(x^{2}\right), \\
& \lim _{k \rightarrow \infty} g\left(g\left(x_{k}^{n}\right)\right)=g\left(x^{n}\right) .
\end{aligned}
$$

From (2.10) and by regarding commutativity of $F$ and $g$

$$
\begin{aligned}
& g\left(g\left(x_{k+1}^{1}\right)\right)=g\left(F\left(x_{k}^{1}, x_{k}^{2}, \ldots, x_{k}^{n}\right)\right)=F\left(g\left(x_{k}^{1}\right), g\left(x_{k}^{2}\right), \ldots, g\left(x_{k}^{n}\right)\right), \\
& g\left(g\left(x_{k+1}^{2}\right)\right)=g\left(F\left(x_{k}^{2}, \ldots, x_{k}^{n}, x_{k}^{1}\right)\right)=F\left(g\left(x_{k}^{2}\right), \ldots, g\left(x_{k}^{n}\right), g\left(x_{k}^{1}\right)\right), \\
& \vdots \\
& g\left(g\left(x_{k+1}^{n}\right)\right)=g\left(F\left(x_{k}^{n}, x_{k}^{1}, \ldots, x_{k}^{n-1}\right)\right)=F\left(g\left(x_{k}^{n}\right), g\left(x_{k}^{1}\right), \ldots, g\left(x_{k}^{n-1}\right)\right) .
\end{aligned}
$$


We shall show that

$$
\begin{aligned}
& F\left(x^{1}, x^{2}, \ldots, x^{n}\right)=g\left(x^{1}\right), \\
& F\left(x^{2}, \ldots, x^{n}, x^{1}\right)=g\left(x^{2}\right), \\
& \vdots \\
& F\left(x^{n}, x^{1}, \ldots, x^{n-1}\right)=g\left(x^{n}\right) .
\end{aligned}
$$

Suppose now, (i) holds. Then by (2.8), (2.32) and (2.30), we have

$$
\begin{aligned}
g\left(x^{1}\right) & =\lim _{k \rightarrow \infty} g\left(g\left(x_{k+1}^{1}\right)\right)=\lim _{k \rightarrow \infty} g\left(F\left(x_{k}^{1}, x_{k}^{2}, \ldots, x_{k}^{n}\right)\right) \\
& =\lim _{k \rightarrow \infty} F\left(g\left(x_{k}^{1}\right), g\left(x_{k}^{2}\right), \ldots, g\left(x_{k}^{n}\right)\right) \\
& =F\left(\lim _{k \rightarrow \infty} g\left(x_{k}^{1}\right), \lim _{k \rightarrow \infty} g\left(x_{k}^{2}\right), \ldots, \lim _{k \rightarrow \infty} g\left(x_{k}^{n}\right)\right) \\
& =F\left(x^{1}, x^{2}, \ldots, x^{n}\right) .
\end{aligned}
$$

Analogously,

$$
\begin{aligned}
g\left(x^{2}\right) & =g\left(g\left(x_{k+1}^{2}\right)\right)=\lim _{k \rightarrow \infty} g\left(F\left(x_{k}^{2}, \ldots, x_{k}^{n}, x_{k}^{1}\right)\right) \\
& =\lim _{k \rightarrow \infty} F\left(g\left(x_{k}^{2}\right), \ldots, g\left(x_{k}^{n}\right), g\left(x_{k}^{1}\right)\right) \\
& =F\left(\lim _{k \rightarrow \infty} g\left(x_{k}^{2}\right), \ldots, \lim _{k \rightarrow \infty} g\left(x_{k}^{n}\right), \lim _{k \rightarrow \infty} g\left(x_{k}^{1}\right)\right) \\
& =F\left(x^{2}, \ldots, x^{n}, x^{1}\right), \\
\vdots & \\
g\left(x^{n}\right) & =g\left(g\left(x_{k+1}^{n}\right)\right)=\lim _{k \rightarrow \infty} g\left(F\left(x^{n}, x^{1}, \ldots, x^{n-1}\right)\right) \\
& =\lim _{k \rightarrow \infty} F\left(g\left(x_{k}^{n}\right), g\left(x_{k}^{1}\right), \ldots, g\left(x_{k}^{n-1}\right)\right) \\
& =F\left(\lim _{k \rightarrow \infty} g\left(x_{k}^{n}\right), \lim _{k \rightarrow \infty} g\left(x_{k}^{1}\right), \ldots, \lim _{k \rightarrow \infty} g\left(x_{k}^{n-1}\right)\right) \\
& =F\left(x^{n}, x^{1}, \ldots, x^{n-1}\right) .
\end{aligned}
$$

Thus, we have

$$
\begin{aligned}
& g\left(x^{1}\right)=F\left(x^{1}, x^{2}, \ldots, x^{n}\right), \\
& g\left(x^{2}\right)=F\left(x^{2}, \ldots, x^{n}, x^{1}\right), \\
& \vdots \\
& g\left(x^{n}\right)=F\left(x^{n}, x^{1}, \ldots, x^{n-1}\right) .
\end{aligned}
$$

Suppose now the assumption (b) holds. If $n$ is odd since $g\left(x_{k}^{1}\right), g\left(x_{k}^{3}\right), \ldots, g\left(x_{k}^{n}\right)$ are nondecreasing and $g\left(x_{k}^{2}\right), g\left(x_{k}^{4}\right), \ldots, g\left(x_{k}^{n-1}\right)$ are non-increasing, if $n$ is even since $g\left(x_{k}^{1}\right), g\left(x_{k}^{3}\right), \ldots$, 
$g\left(x_{k}^{n-1}\right)$ are non-decreasing and $g\left(x_{k}^{2}\right), g\left(x_{k}^{4}\right), \ldots, g\left(x_{k}^{n}\right)$ are non-increasing and by considering $g\left(x_{k}^{1}\right) \rightarrow x^{1}, g\left(x_{k}^{2}\right) \rightarrow x^{2}, \ldots, g\left(x_{k}^{n}\right) \rightarrow x^{n}$ we have

$$
\begin{aligned}
& \left.g\left(x_{k}^{1}\right) \geq x^{1}, \quad g\left(x_{k}^{2}\right) \leq x^{2}, \quad \ldots, \quad g\left(x_{k}^{n}\right) \leq x^{n} \quad \text { (if } n \text { is odd }\right), \\
& g\left(x_{k}^{n}\right) \geq x^{n} \quad(\text { if } n \text { is even })
\end{aligned}
$$

for all $k$. Thus, by triangle inequality and (2.32)

$$
\begin{aligned}
& d\left(g\left(x^{1}\right), F\left(x^{1}, x^{2}, \ldots, x^{n}\right)\right) \\
& \leq d\left(g\left(x^{1}\right), g\left(g\left(x_{k+1}^{1}\right)\right)\right) \\
& \quad+d\left(g\left(g\left(x_{k+1}^{1}\right), F\left(x^{1}, x^{2}, \ldots, x^{n}\right)\right)\right) \\
& \leq d\left(g\left(x^{1}\right), g\left(g\left(x_{k+1}^{1}\right)\right)\right) \\
& \quad+\phi\left(\frac { 1 } { n } \left[d\left(g\left(g\left(x_{k}^{1}\right)\right), g\left(x^{1}\right)\right)+d\left(g\left(g\left(x_{k}^{2}\right)\right), g\left(x^{2}\right)\right)\right.\right. \\
& \left.\left.\quad+\cdots+d\left(g\left(g\left(x_{k}^{n}\right)\right), g\left(x^{n}\right)\right)\right]\right) .
\end{aligned}
$$

Letting $k \rightarrow \infty$ implies that $d\left(g\left(x^{1}\right), F\left(x^{1}, x^{2}, \ldots, x^{n}\right)\right) \leq 0$. Hence, $g\left(x^{1}\right)=F\left(x^{1}, x^{2}, \ldots, x^{n}\right)$. Analogously, we can get that

$$
g\left(x^{2}\right)=F\left(x^{2}, \ldots, x^{n}, x^{1}\right), \quad \ldots, \quad g\left(x^{n}\right)=F\left(x^{n}, x^{1}, \ldots, x^{n-1}\right) .
$$

Thus, we proved that $F$ and $g$ have a $n$-tuplet coincidence point.

Corollary 1 The above theorem reduces to Theorem 2.1 of [2] for $n=1$ and $g(x)=x$ if (i) is satisfied and $\phi(t)=$ ct where $c \in(0,1)$.

The following corollary is a generalization of Corollary $2.1 \mathrm{in} \mathrm{[11]} \mathrm{and} \mathrm{Theorem} 2.1 \mathrm{in}$ [4].

Corollary 2 Let $(X, \leq)$ be a partially ordered set and suppose that $(X, d)$ is complete metric space. Suppose $F: X^{n} \rightarrow X$ and there exist $\phi \in \Phi$ such that $F$ has the mixed g-monotone property and there exist a $m \in[0,1)$ with

$$
\begin{aligned}
& \phi\left(F\left(x^{1}, x^{2}, \ldots, x^{n}\right), F\left(y^{1}, y^{2}, \ldots, y^{n}\right)\right) \\
& \quad \leq \frac{m}{n}\left[d\left(g\left(x^{1}\right), g\left(y^{1}\right)\right)+d\left(g\left(x^{2}\right), g\left(y^{2}\right)\right)+\cdots+d\left(g\left(x^{n}\right), g\left(y^{n}\right)\right)\right]
\end{aligned}
$$

for all $x^{1}, x^{2}, \ldots, x^{n}, y^{1}, y^{2}, \ldots, y^{n} \in X$ for which $g\left(x^{1}\right) \leq g\left(y^{1}\right), g\left(x^{2}\right) \geq g\left(y^{2}\right), \ldots, g\left(x^{n}\right) \leq g\left(y^{n}\right)$ (if $n$ is odd), $g\left(x^{n}\right) \geq g\left(y^{n}\right)$ (if $n$ is even). Assume also Condition 1 holds, and assume that $F\left(X^{n}\right) \subset g(X), g$ is continuous and commutes with $F$. If there exist $z^{1}, z^{2}, \ldots, z^{n} \in X$ such 
that

$$
\begin{aligned}
& g\left(z^{1}\right) \leq F\left(z^{1}, z^{2}, \ldots, z^{n}\right), \\
& g\left(z^{2}\right) \geq F\left(z^{2}, \ldots, z^{n}, z^{1}\right), \\
& \vdots \\
& g\left(z^{n}\right) \leq F\left(z^{n}, z^{1}, \ldots, z^{n-1}\right) \quad(\text { if } n \text { is odd }), \\
& g\left(z^{n}\right) \geq F\left(z^{n}, z^{1}, \ldots, z^{n-1}\right) \quad(\text { if } n \text { is even })
\end{aligned}
$$

then there exist $x^{1}, x^{2}, \ldots, x^{n} \in X$ such that

$$
\begin{aligned}
& g\left(x^{1}\right)=F\left(x^{1}, x^{2}, \ldots, x^{n}\right), \\
& g\left(x^{2}\right)=F\left(x^{2}, \ldots, x^{n}, x^{1}\right), \\
& \vdots \\
& g\left(x^{n}\right)=F\left(x^{n}, x^{1}, \ldots, x^{n-1}\right),
\end{aligned}
$$

that is, $F$ and $g$ have a $n$-tuplet coincidence point.

Proof It is sufficient to take $\phi=m t$ with $m \in[0,1)$ in previous theorem.

\section{Uniqueness of $\boldsymbol{n}$-tuplet fixed point}

For all $\left(x^{1}, x^{2}, \ldots, x^{n}\right),\left(y^{1}, y^{2}, \ldots, y^{n}\right) \in X^{n}$,

$$
\begin{aligned}
& \left(x^{1}, x^{2}, \ldots, x^{n}\right) \leq\left(y^{1}, y^{2}, \ldots, y^{n}\right) \\
& \Longleftrightarrow \quad x^{1} \leq y^{1}, \quad x^{2} \geq y^{2}, \quad \ldots, \quad x^{n} \leq y^{n} \quad(\text { if } n \text { is odd }), \\
& \left.x^{n} \geq y^{n} \quad \text { (if } n \text { is even }\right) .
\end{aligned}
$$

We say that $\left(x^{1}, x^{2}, \ldots, x^{n}\right)$ is equal to $\left(y^{1}, y^{2}, \ldots, y^{n}\right)$ if and only if $x^{1}=y^{1}, x^{2}=y^{2}, \ldots, x^{n}=y^{n}$.

Theorem 2 In addition to hypothesis Theorem 1, assume that for all $\left(x^{1}, x^{2}, \ldots, x^{n}\right)$, $\left(y^{1}, y^{2}, \ldots, y^{n}\right) \in X^{n}$ there exist $\left(z^{1}, z^{2}, \ldots, z^{n}\right) \in X^{n}$ such that

$$
\left(F\left(z^{1}, z^{2}, \ldots, z^{n}\right), F\left(z^{2}, \ldots, z^{n}, z^{1}\right), \ldots, F\left(z^{n}, z^{1}, \ldots, z^{n-1}\right)\right)
$$

is comparable to

$$
\left(F\left(x^{1}, x^{2}, \ldots, x^{n}\right), F\left(x^{2}, \ldots, x^{n}, x^{1}\right), \ldots, F\left(x^{n}, x^{1}, \ldots, x^{n-1}\right)\right)
$$

and

$$
\left(F\left(y^{1}, y^{2}, \ldots, y^{n}\right), F\left(y^{2}, \ldots, y^{n}, y^{1}\right), \ldots, F\left(y^{n}, y^{1}, \ldots, y^{n-1}\right)\right)
$$


Then F and $g$ have a unique $n$-tuplet common fixed point, that is, there exist $\left(x^{1}, x^{2}, \ldots, x^{n}\right) \in$ $X^{n}$ such that

$$
\begin{aligned}
& x^{1}=g\left(x^{1}\right)=F\left(x^{1}, x^{2}, \ldots, x^{n}\right), \\
& x^{2}=g\left(x^{2}\right)=F\left(x^{2}, \ldots, x^{n}, x^{1}\right), \\
& \vdots \\
& x^{n}=g\left(x^{n}\right)=F\left(x^{n}, x^{1}, \ldots, x^{n-1}\right) .
\end{aligned}
$$

Proof From the Theorem 1, the set of $n$-tuplet coincidences is non-empty. We will show that if $\left(x^{1}, x^{2}, \ldots, x^{n}\right)$ and $\left(y^{1}, y^{2}, \ldots, y^{n}\right)$ are $n$-tuplet coincidence points, that is, if

$$
\begin{aligned}
& g\left(x^{1}\right)=F\left(x^{1}, x^{2}, \ldots, x^{n}\right), \\
& g\left(x^{2}\right)=F\left(x^{2}, \ldots, x^{n}, x^{1}\right), \\
& \vdots \\
& g\left(x^{n}\right)=F\left(x^{n}, x^{1}, \ldots, x^{n-1}\right)
\end{aligned}
$$

and

$$
\begin{aligned}
& g\left(y^{1}\right)=F\left(y^{1}, y^{2}, \ldots, y^{n}\right), \\
& g\left(y^{2}\right)=F\left(y^{2}, \ldots, y^{n}, y^{1}\right), \\
& \vdots \\
& g\left(y^{n}\right)=F\left(y^{n}, y^{1}, \ldots, y^{n-1}\right),
\end{aligned}
$$

then

$$
\begin{aligned}
& g\left(x^{1}\right)=g\left(y^{1}\right), \\
& g\left(x^{2}\right)=g\left(y^{2}\right), \\
& \vdots \\
& g\left(x^{n}\right)=g\left(y^{n}\right) .
\end{aligned}
$$

By assumption there is $\left(z^{1}, z^{2}, \ldots, z^{n}\right) \in X^{n}$ such that

$$
\left(F\left(z^{1}, z^{2}, \ldots, z^{n}\right), F\left(z^{2}, \ldots, z^{n}, z^{1}\right), \ldots, F\left(z^{n}, z^{1}, \ldots, z^{n-1}\right)\right)
$$

is comparable with

$$
\left(F\left(x^{1}, x^{2}, \ldots, x^{n}\right), F\left(x^{2}, \ldots, x^{n}, x^{1}\right), \ldots, F\left(x^{n}, x^{1}, \ldots, x^{n-1}\right)\right)
$$

and

$$
\left(F\left(y^{1}, y^{2}, \ldots, y^{n}\right), F\left(y^{2}, \ldots, y^{n}, y^{1}\right), \ldots, F\left(y^{n}, y^{1}, \ldots, y^{n-1}\right)\right) .
$$


Define sequences $\left(g\left(z_{k}^{1}\right)\right),\left(g\left(z_{k}^{2}\right)\right), \ldots,\left(g\left(z_{k}^{n}\right)\right)$ such that $z^{1}=z_{0}^{1}, z^{2}=z_{0}^{2}, \ldots, z^{n}=z_{0}^{n}$ and

$$
\begin{aligned}
& g\left(z_{k}^{1}\right)=F\left(z_{k-1}^{1}, z_{k-1}^{2}, \ldots, z_{k-1}^{n}\right), \\
& g\left(z_{k}^{2}\right)=F\left(z_{k-1}^{2}, \ldots, z_{k-1}^{n}, z_{k-1}^{2}\right), \\
& \vdots \\
& g\left(z_{k}^{n}\right)=F\left(z_{k-1}^{n}, z_{k-1}^{1}, \ldots, z_{k-1}^{n-1}\right) .
\end{aligned}
$$

Since (3.4) and (3.5) comparable with (3.3), we may assume that

$$
\left(g\left(x^{1}\right), g\left(x^{2}\right), \ldots, g\left(x^{n}\right)\right) \geq\left(g\left(z^{1}\right), g\left(z^{2}\right), \ldots, g\left(z^{n}\right)\right)=\left(g\left(z_{0}^{1}\right), g\left(z_{0}^{2}\right), \ldots, g\left(z_{0}^{n}\right)\right) .
$$

By using (2.11), we get that

$$
\left(g\left(x^{1}\right), g\left(x^{2}\right), \ldots, g\left(x^{n}\right)\right) \geq\left(g\left(z_{k}^{1}\right), g\left(z_{k}^{2}\right), \ldots, g\left(z_{k}^{n}\right)\right)
$$

for all $k$. From (3.1), we have

$$
\begin{aligned}
& g\left(x^{1}\right) \leq g\left(z_{k}^{1}\right), \\
& g\left(x^{2}\right) \geq g\left(z_{k}^{2}\right), \\
& \vdots \\
& g\left(x^{n}\right) \leq g\left(z_{k}^{n}\right) \quad \text { (if } n \text { is odd), } \\
& g\left(x^{n}\right) \geq g\left(z_{k}^{n}\right) \quad \text { (if } n \text { is even). }
\end{aligned}
$$

By (3.7) and (2.6), we have

$$
\begin{aligned}
& d\left(g\left(x^{1}\right), g\left(z_{k+1}^{1}\right)\right) \\
& =d\left(F\left(x^{1}, x^{2}, \ldots, x^{n}\right), F\left(z_{k}^{1}, z_{k}^{2}, \ldots, z_{k}^{n}\right)\right) \\
& \leq \phi\left(\frac{1}{n}\left[d\left(g\left(x^{1}\right), g\left(z_{k}^{1}\right)\right)+d\left(g\left(x^{2}\right), g\left(z_{k}^{2}\right)\right)+\cdots+d\left(g\left(x^{n}\right), g\left(z_{k}^{n}\right)\right)\right]\right), \\
& d\left(g\left(x^{2}\right), g\left(z_{k+1}^{2}\right)\right) \\
& =d\left(F\left(x^{2}, \ldots, x^{n}, x^{1}\right), F\left(z_{k}^{2}, \ldots, z_{k}^{n}, z_{k}^{1}\right)\right) \\
& \leq \phi\left(\frac{1}{n}\left[d\left(g\left(x^{2}\right), g\left(z_{k}^{2}\right)\right)+\cdots+d\left(g\left(x^{n}\right), g\left(z_{k}^{n}\right)\right)+d\left(g\left(x^{1}\right), g\left(z_{k}^{1}\right)\right)\right]\right), \\
& d\left(g\left(x^{n}\right), g\left(z_{k+1}^{n}\right)\right) \\
& =d\left(F\left(x^{n}, x^{1}, \ldots, x^{n-1}\right), F\left(z_{k}^{n}, z_{k}^{1}, \ldots, z_{k}^{n-1}\right)\right) \\
& \leq \phi\left(\frac{1}{n}\left[d\left(g\left(x^{n}\right), g\left(z_{k}^{n}\right)\right)+d\left(g\left(x^{1}\right), g\left(z_{k}^{1}\right)\right)+\cdots+d\left(g\left(x^{n-1}\right), g\left(z_{k}^{n-1}\right)\right)\right]\right) .
\end{aligned}
$$


Adding (3.8)-(3.10), we get

$$
\begin{aligned}
& \frac{d\left(g\left(x^{1}\right), g\left(z_{k+1}^{1}\right)\right)+d\left(g\left(x^{2}\right), g\left(z_{k+1}^{2}\right)\right)+\cdots+d\left(g\left(x^{n}\right), g\left(z_{k+1}^{n}\right)\right)}{n} \\
& \quad \leq \phi\left(\frac{1}{n}\left[d\left(g\left(x^{2}\right), g\left(z_{k}^{2}\right)\right)+\cdots+d\left(g\left(x^{n}\right), g\left(z_{k}^{n}\right)\right)+d\left(g\left(x^{1}\right), g\left(z_{k}^{1}\right)\right)\right]\right) .
\end{aligned}
$$

Hence, it follows

$$
\begin{aligned}
& \frac{d\left(g\left(x^{1}\right), g\left(z_{k+1}^{1}\right)\right)+d\left(g\left(x^{2}\right), g\left(z_{k+1}^{2}\right)\right)+\cdots+d\left(g\left(x^{n}\right), g\left(z_{k+1}^{n}\right)\right)}{n} \\
& \quad \leq \phi^{k}\left(\frac{1}{n}\left[d\left(g\left(x^{2}\right), g\left(z_{k}^{2}\right)\right)+\cdots+d\left(g\left(x^{n}\right), g\left(z_{k}^{n}\right)\right)+d\left(g\left(x^{1}\right), g\left(z_{k}^{1}\right)\right)\right]\right)
\end{aligned}
$$

for each $k \geq 1$. It is known that $\phi(t)<t$ and $\lim _{r \rightarrow t^{+}} \phi(r)<t$ imply $\lim _{k \rightarrow \infty} \phi^{k}(t)=0$ for each $t>0$. Thus, from (3.12)

$$
\begin{aligned}
& \lim _{k \rightarrow \infty} d\left(g\left(x^{1}\right), g\left(z_{k+1}^{1}\right)\right)=0, \\
& \lim _{k \rightarrow \infty} d\left(g\left(x^{2}\right), g\left(z_{k+1}^{2}\right)\right)=0, \\
& \vdots \\
& \lim _{k \rightarrow \infty} d\left(g\left(x^{2}\right), g\left(z_{k+1}^{2}\right)\right)=0 .
\end{aligned}
$$

Analogously, we can show that

$$
\begin{aligned}
& \lim _{k \rightarrow \infty} d\left(g\left(y^{1}\right), g\left(z_{k+1}^{1}\right)\right)=0, \\
& \lim _{k \rightarrow \infty} d\left(g\left(y^{2}\right), g\left(z_{k+1}^{2}\right)\right)=0, \\
& \vdots \\
& \lim _{k \rightarrow \infty} d\left(g\left(y^{2}\right), g\left(z_{k+1}^{2}\right)\right)=0 .
\end{aligned}
$$

Combining (3.13) and (3.14) and by using the triangle inequality

$$
\begin{aligned}
& d\left(g\left(x^{1}\right), g\left(y^{1}\right)\right) \leq d\left(g\left(x^{1}\right), g\left(z_{k+1}^{1}\right)\right)+d\left(g\left(y^{1}\right), g\left(z_{k+1}^{1}\right)\right) \rightarrow 0 \quad \text { as } k \rightarrow \infty, \\
& d\left(g\left(x^{2}\right), g\left(y^{2}\right)\right) \leq d\left(g\left(x^{2}\right), g\left(z_{k+1}^{2}\right)\right)+d\left(g\left(y^{2}\right), g\left(z_{k+1}^{2}\right)\right) \rightarrow 0 \quad \text { as } k \rightarrow \infty, \\
& \vdots \\
& d\left(g\left(x^{n}\right), g\left(y^{n}\right)\right) \leq d\left(g\left(x^{n}\right), g\left(z_{k+1}^{n}\right)\right)+d\left(g\left(y^{n}\right), g\left(z_{k+1}^{n}\right)\right) \rightarrow 0 \quad \text { as } k \rightarrow \infty .
\end{aligned}
$$

Hence, we get $g\left(x^{1}\right)=g\left(y^{1}\right), g\left(x^{2}\right)=g\left(y^{2}\right), \ldots, g\left(x^{n}\right)=g\left(y^{n}\right)$. Thus, we proved claim of theorem. 
By commutativity of $F$ and $g$,

$$
\begin{aligned}
& g\left(g\left(x^{1}\right)\right)=g\left(F\left(x^{1}, x^{2}, \ldots, x^{n}\right)\right)=F\left(g\left(x^{1}\right), g\left(x^{2}\right), \ldots, g\left(x^{n}\right)\right), \\
& g\left(g\left(x^{2}\right)\right)=g\left(F\left(x^{2}, \ldots, x^{n}, x^{1}\right)\right)=F\left(g\left(x^{2}\right), \ldots, g\left(x^{n}\right), g\left(x^{1}\right)\right), \\
& \vdots \\
& g\left(g\left(x^{n}\right)\right)=g\left(F\left(x^{n}, x^{1}, \ldots, x^{n-1}\right)\right)=F\left(g\left(x^{n}\right), g\left(x^{1}\right), \ldots, g\left(x^{n-1}\right)\right) .
\end{aligned}
$$

Denote $g\left(x^{1}\right)=w^{1}, g\left(x^{2}\right)=w^{2}, \ldots, g\left(x^{n}\right)=w^{n}$. Since (3.16), we get

$$
\begin{aligned}
& g\left(w^{1}\right)=F\left(w^{1}, w^{2}, \ldots, w^{n}\right), \\
& g\left(w^{2}\right)=F\left(w^{2}, \ldots, w^{n}, w^{1}\right), \\
& \vdots \\
& g\left(w^{n}\right)=F\left(w^{n}, w^{1}, \ldots, w^{n-1}\right) .
\end{aligned}
$$

Thus, $\left(w^{1}, w^{2}, \ldots, w^{n}\right)$ is a $n$-tuplet coincidence point. Then from assumption in theorem with $y^{1}=w^{1}, \ldots, y^{n}=w^{n}$ it follows $g\left(w^{1}\right)=g\left(x^{1}\right), g\left(w^{2}\right)=g\left(x^{2}\right), \ldots, g\left(w^{n}\right)=g\left(x^{n}\right)$, that is

$$
g\left(w^{1}\right)=w^{1}, \quad \ldots, \quad g\left(w^{n}\right)=w^{n} .
$$

From (3.18) and (3.17),

$$
\begin{aligned}
& w^{1}=g\left(w^{1}\right)=F\left(w^{1}, w^{2}, \ldots, w^{n}\right), \\
& w^{2}=g\left(w^{2}\right)=F\left(w^{2}, \ldots, w^{n}, w^{1}\right), \\
& \vdots \\
& w^{n}=g\left(w^{n}\right)=F\left(w^{n}, w^{1}, \ldots, w^{n-1}\right) .
\end{aligned}
$$

Therefore, $\left(w^{1}, w^{2}, \ldots, w^{n}\right)$ is $n$-tuplet common fixed point of $F$ and $g$. To prove the uniqueness, assume that $\left(u^{1}, u^{2}, \ldots, u^{n}\right)$ is another $n$-tuplet common fixed point. Then by assumption in theorem we have

$$
\begin{aligned}
& u^{1}=g\left(u^{1}\right)=g\left(w^{1}\right), \\
& u^{2}=g\left(u^{2}\right)=g\left(w^{2}\right), \\
& \vdots \\
& u^{n}=g\left(u^{n}\right)=g\left(w^{n}\right) .
\end{aligned}
$$

Corollary 3 Let $(X, \leq)$ be partially ordered set and suppose that $(X, d)$ is complete metric space. Suppose $F: X^{n} \rightarrow X$ and there exist $\phi \in \Phi$ such that $F$ has the mixed $g$-monotone 
property and

$$
\begin{aligned}
& d\left(F\left(x^{1}, x^{2}, x^{3}, \ldots, x^{n}\right), F\left(y^{1}, y^{2}, y^{3}, \ldots, y^{n}\right)\right) \\
& \quad \leq \phi\left(\frac{d\left(g\left(x^{1}\right), g\left(y^{1}\right)\right)+d\left(g\left(x^{2}\right), g\left(y^{2}\right)\right)+\cdots+d\left(g\left(x^{n}\right), g\left(y^{n}\right)\right)}{n}\right)
\end{aligned}
$$

for all $x^{1}, x^{2}, x^{3}, \ldots, x^{n}, y^{1}, y^{2}, y^{3}, \ldots, y^{n} \in X$ for which $x^{1} \leq y^{1}, x^{2} \geq y^{2}, \ldots, x^{n} \leq y^{n}$ (ifn is odd), $x^{n} \geq y^{n}$ (if $n$ is even). Suppose there exist $x_{0}^{1}, x_{0}^{2}, x_{0}^{3}, \ldots, x_{0}^{n} \in X$ such that

$$
\begin{aligned}
& x_{0}^{1} \leq F\left(x_{0}^{1}, x_{0}^{2}, x_{0}^{3}, \ldots, x_{0}^{n}\right), \\
& x_{0}^{2} \geq F\left(x_{0}^{2}, x_{0}^{3}, \ldots, x_{0}^{n}, x_{0}^{1}\right), \\
& x_{0}^{n} \leq F\left(x_{0}^{n}, x_{0}^{1}, x_{0}^{2}, \ldots, x_{0}^{n-1}\right) \quad(\text { if } n \text { is odd }), \\
& \vdots \\
& x_{0}^{n} \geq F\left(x_{0}^{n}, x_{0}^{1}, x_{0}^{2}, \ldots, x_{0}^{n-1}\right) \quad(\text { if } n \text { is even }) .
\end{aligned}
$$

Assume also that Condition 1 holds. Then there exist $x^{1}, x^{2}, x^{3}, \ldots, x^{n}$ such that

$$
\begin{aligned}
& x^{1}=F\left(x^{1}, x^{2}, x^{3}, \ldots, x^{n}\right), \\
& x^{2}=F\left(x^{2}, x^{3}, \ldots, x^{n}, x^{1}\right), \\
& \vdots \\
& x^{n}=F\left(x^{n}, x^{1}, x^{2}, \ldots, x^{n-1}\right) .
\end{aligned}
$$

That is $F$ has a n-tuplet fixed point.

Proof Take $g(x)=x$, then the assumption in Theorem 1 are satisfied. Thus, we get the result.

Corollary 4 Let $(X, \leq)$ be partially ordered set and suppose that $(X, d)$ is complete metric space. Suppose $F: X^{n} \rightarrow X$ and there exist $\phi \in \Phi$ such that $F$ has the mixed $g$-monotone property and there exist $m \in[0,1)$ with

$$
\begin{aligned}
& \phi\left(F\left(x^{1}, x^{2}, \ldots, x^{n}\right), F\left(y^{1}, y^{2}, \ldots, y^{n}\right)\right) \\
& \quad \leq \frac{m}{n}\left[d\left(g\left(x^{1}\right), g\left(y^{1}\right)\right)+d\left(g\left(x^{2}\right), g\left(y^{2}\right)\right)+\cdots+d\left(g\left(x^{n}\right), g\left(y^{n}\right)\right)\right]
\end{aligned}
$$

for all $x^{1}, x^{2}, \ldots, x^{n}, y^{1}, y^{2}, \ldots, y^{n} \in X$ for which $x^{1} \leq y^{1}, x^{2} \geq y^{2}, \ldots, x^{n} \leq y^{n}$ (if $n$ is odd), $x^{n} \geq y^{n}$ (if $n$ is even). Suppose there exist $x_{0}^{1}, x_{0}^{2}, x_{0}^{3}, \ldots, x_{0}^{n} \in X$ such that

$$
\begin{aligned}
& x_{0}^{1} \leq F\left(x_{0}^{1}, x_{0}^{2}, x_{0}^{3}, \ldots, x_{0}^{n}\right), \\
& x_{0}^{2} \geq F\left(x_{0}^{2}, x_{0}^{3}, \ldots, x_{0}^{n}, x_{0}^{1}\right),
\end{aligned}
$$




$$
\begin{array}{ll}
x_{0}^{n} \leq F\left(x_{0}^{n}, x_{0}^{1}, x_{0}^{2}, \ldots, x_{0}^{n-1}\right) & (\text { if } n \text { is odd }), \\
x_{0}^{n} \geq F\left(x_{0}^{n}, x_{0}^{1}, x_{0}^{2}, \ldots, x_{0}^{n-1}\right) & (\text { if } n \text { is even }) .
\end{array}
$$

Assume also that Condition 1 holds. Then there exist $x^{1}, x^{2}, x^{3}, \ldots, x^{n}$ such that

$$
\begin{aligned}
& x^{1}=F\left(x^{1}, x^{2}, x^{3}, \ldots, x^{n}\right), \\
& x^{2}=F\left(x_{0}^{2}, x_{0}^{3}, \ldots, x_{0}^{n}, x_{0}^{1}\right), \\
& \vdots \\
& x^{n}=F\left(x^{n}, x^{1}, x^{2}, \ldots, x^{n-1}\right) .
\end{aligned}
$$

\section{That is $F$ and $g$ have $n$-tuplet coincidence point.}

Proof Taking $\phi(t)=m \cdot t$ with $m \in[0,1)$ in above corollary we obtain this corollary.

\section{Competing interests}

The authors declare that they have no competing interests.

\section{Author details}

'Department of Mathematics, The Faculty of Arts and Sciences, Yildiz Technical University, Davutpasa Campus, Esenler, Istanbul, 34210, Turkey. ${ }^{2}$ Current address: Department of Mathematical Engineering, Yildiz Technical University, Davutpasa Campus, Esenler, Istanbul, 34220, Turkey.

\section{Acknowledgements}

This work is supported by Yildiz Technical University Scientific Research Projects Coordination Unit under the project number BAPK 2012-07-03-DOP03.

\section{Received: 4 January 2013 Accepted: 5 April 2013 Published: 22 April 2013}

\section{References}

1. Banach, S: Sur les operations dans les ensembles abstraits et leur applications aux equations integrales. Fundam. Math. 3, 133-181 (1922)

2. Ran, ACM, Reurings, MCB: A fixed point theorem in partially ordered sets and some applications to matrix equations. Proc. Am. Math. Soc. 132(5), 1435-1443 (2004). doi:10.1090/s0002-9939-03-07220-4

3. Nieto, J, Rodríguez-López, R: Contractive mapping theorems in partially ordered sets and applications to ordinary differential equations. Order 22(3), 223-239 (2005). doi:10.1007/s11083-005-9018-5

4. Bhaskar, TG, Lakshmikantham, V: Fixed point theorems in partially ordered metric spaces and applications. Nonlinear Anal., Theory Methods Appl. 65(7), 1379-1393 (2006). doi:10.1016/j.na.2005.10.017

5. Abbas, M, Khan, MA, Radenovic, S: Common coupled fixed point theorems in cone metric spaces for $w$-compatible mappings. Appl. Math. Comput. 217(1), 195-202 (2010). doi:10.1016/j.amc.2010.05.042

6. Abbas, M, Damjanovic, B, Lazovic, R: Fuzzy common fixed point theorems for generalized contractive mappings. Appl. Math. Lett. 23(11), 1326-1330 (2010). doi:10.1016/j.aml.2010.06.023

7. Aydi, H, Karapinar, E, Shatanawi, W: Coupled fixed point results for $(\psi, \phi)$-weakly contractive condition in ordered partial metric spaces. Comput. Math. Appl. 62(12), 4449-4460 (2011). doi:10.1016/j.camwa.2011.10.021

8. Berinde, $\vee$ : Generalized coupled fixed point theorems for mixed monotone mappings in partially ordered metric spaces. Nonlinear Anal., Theory Methods Appl. 74(18), 7347-7355 (2011). doi:10.1016/j.na.2011.07.053

9. Berinde, $\vee$ : Coupled coincidence point theorems for mixed monotone nonlinear operators. Comput. Math. Appl. 64(6), 1770-1777 (2012). doi:10.1016/j.camwa.2012.02.012

10. Gordji, ME, Ghods, S, Ghods, V, Hadian, M: Coupled fixed point theorem for generalized fuzzy Meir-Keeler contraction in fuzzy metric spaces. J. Comput. Anal. Appl. 14(2), 271-277 (2012)

11. Lakshmikantham, V, Ciric, L: Coupled fixed point theorems for nonlinear contractions in partially ordered metric spaces. Nonlinear Anal., Theory Methods Appl. 70(12), 4341-4349 (2009). doi:10.1016/j.na.2008.09.020

12. Berinde, $V$, Borcut, $M$ : Tripled fixed point theorems for contractive type mappings in partially ordered metric spaces. Nonlinear Anal., Theory Methods Appl. 74(15), 4889-4897 (2011). doi:10.1016/j.na.2011.03.032

13. Aydi, H, Karapınar, E, Yüce, IS: Quadruple fixed point theorems in partially ordered metric spaces depending on another function. ISRN Appl. Math. (2012). doi:10.5402/2012/539125

14. Karapinar, E, Shatanawi, W, Mustafa, Z: Quadruple fixed point theorems under nonlinear contractive conditions in partially ordered metric spaces. J. Appl. Math. (2012). doi:10.1155/2012/951912

15. Mustafa, Z, Aydi, H, Karapinar, E: Mixed g-monotone property and quadruple fixed point theorems in partially ordered metric spaces. Fixed Point Theory Appl. 2012 1-19 (2012). doi:10.1186/1687-1812-2012-71

16. Karapinar, E, Berinde, V: Quadruple fixed point theorems for nonlinear contractions in partially ordered metric spaces. Banach J. Math. Anal. 6(1), 74-89 (2012) 
doi:10.1186/1029-242X-2013-196

Cite this article as: Ertürk and Karakaya: $n$-tuplet fixed point theorems for contractive type mappings in partially ordered metric spaces. Journal of Inequalities and Applications 2013 2013:196.

Submit your manuscript to a SpringerOpen ${ }^{\circ}$ journal and benefit from:

- Convenient online submission

- Rigorous peer review

- Immediate publication on acceptance

Open access: articles freely available online

- High visibility within the field

- Retaining the copyright to your article

Submit your next manuscript at $\boldsymbol{s p r i n g e r o p e n . c o m ~}$ 